\title{
String Tension and Glueball Mass in a Lattice Theory of Disconnected, Selfintersecting Random Surfaces
}

\author{
Robert Schrader $\star$ \\ RIMS, Kyoto University, Kyoto 606, Japan
}

\begin{abstract}
We exhibit the analytic structure of a model of disconnected, selfintersecting random surfaces. This model is shown to have features attractive for a Monte Carlo simulation. Previously obtained numerical data show evidence that for a 3-dim embedding space the string tension vanishes above a critical temperature and has a critical exponent between 1 and 2 . The glueball mass is shown to be bounded below by the mass-gap of the 3-dim Ising model.
\end{abstract}

\section{Introduction}

In a recent article [Sch], we presented some numerical analysis of a lattice theory of disconnected, selfintersecting random surfaces embedded in $\mathbb{R}^{d=3}$. (For motivation of and references to similar and alternative lattice approaches to random surfaces, see [Sch] as well as [DFJ, F].) It is the aim of this paper to continue the analytic discussion of this model. Allowing the embedding space to have arbitrary dimension $d \geqq 3$, we prove existence and properties of the string tension. Comparison with the numerical results obtained in [Sch] indicates that for $d=3$ the string tension vanishes below a critical temperature and goes to zero at the critical temperature with a critical exponent between 1 and 2 . The model also allows for a definition of the glueball mass. Exploiting the isomorphy to the $d=3$ Ising model in 3 dimensions, whereby these surfaces correspond to the Peierls interfaces, we show that the glueball mass is bounded below by the mass gap for the $d=3$ Ising model. These results are obtained by showing that in this model all quantities of interest may be written as (quotients of) Green's functions in the Gibbs ensemble consisting of manifolds without boundary. This makes these quantities easily accessible to an efficient Monte-Carlo computer simulation. For example, it is our impression that the new microcanonical algorithm invented by M. Creutz could be applied.

* On leave of absence from Freie Universität Berlin 
In Sect. 2 we give the definition of the model. We establish the basic analytic properties including physical positivity [OS] (also called reflection positivity, see e.g. [GJ] for a survey). Combined with translation invariance, this allows the application of the well known techniques involving the transfer matrix. In Sect. 3 we briefly recall the equivalence to the Ising model for the case $d=3$ and the $Z_{2}$ lattice gauge theory for arbitrary $d$. Thus most, but not all of our results could be obtained using the equivalence to the $Z_{2}$ lattice gauge theory. Nevertheless, we choose to work out the manifold properties in an intrinsic way, in particular, since our methods easily extend to more complicated models given in the last 2 sections. In Sect. 4 we introduce the string tension and the glueball mass. We establish and discuss their properties. In Sect. 5 we briefly indicate how to extend our analytic discussion to the case, where the manifold is allowed to fold onto itself. Section 6 gives a lattice formulation where in addition the surfaces carry a Yang-Mills field. In an appendix we prove the ergodicity of the Monte-Carlo upgrading procedure used in e.g. [SG] and [Sch].

\section{Analytic Properties of the Model}

Let $(a \mathbb{Z})^{d} \subset \mathbb{R}^{d}$ be a hypercubical lattice. The lattice distance $a>0$ will be arbitrary but fixed. $c^{i}$ will denote any open $i$-cell (vertices, bonds, plaquettes ...) with $i$-dim volume $a^{i}$. The openness condition is for technical convenience. Simultaneously, we make the $c^{2}$ 's into dynamical variables taking values in $\mathbb{Z}_{2}\left(c^{2}=0,1\right)$. We form the groups

and

$$
\mathscr{G}=\prod_{c^{2}} \mathbb{Z}_{2}
$$

$$
\mathscr{G}_{\Lambda}=\prod_{c^{2} \in A} \mathbb{Z}_{2},
$$

where $\Lambda$ is any box in $\mathbb{R}^{d}$. Each $\mathscr{G}_{\Lambda}$ may be viewed as a subgroup of $\mathscr{G}$ or $\mathscr{G}_{A^{\prime}}$ $\left(\Lambda \subseteq \Lambda^{\prime}\right)$. Note to each group element $g=\left\{g\left(c^{2}\right)\right\}_{c^{2}} \in \mathscr{G}$ we associate a "manifold" $M=M(g)$ defined to be the union of the different $c^{2}$ 's for which $g\left(c^{2}\right)=1$. Conversely any such union $M$ of 2-cells $c^{2}$ defines a unique group element $g$. Finally, we give any $c^{2}$ another interpretation, namely as a function on the set of all manifolds, by

$$
c^{2}(M)=\left\{\begin{array}{lll}
1 & \text { if } & c^{2} \in M \\
0 & \text { if } & c^{2} \notin M
\end{array}\right.
$$

We will make free and interchangeable use of these four definitions for any $c^{2}$, namely (i) as a label for a 2-cell, (ii) as the set of points in this 2-cell, (iii) as a dynamical variable taking values 0 and 1 [our previous $g\left(c^{2}\right)$ ] and (iv) as a function on the set of all manifolds in the sense of rel. (3). Next we introduce the symmetric sum of two manifolds $M_{1}$ and $M_{2}$ by

$$
M_{1}+M_{2}=M_{1} \cup M_{2} \backslash M_{1} \cap M_{2} .
$$

Under this sum the manifolds form an abelian group, the empty set being the zero element and all other elements having order 2 . In terms of the above correspondence $M=M(g)$ this group is isomorphic to $\mathscr{G}$ and the $M$ 's with $M \subset A$ form a 
group isomorphic to $\mathscr{G}_{A}$. Also

$$
c^{2}\left(M_{1}+M_{2}\right) \equiv c^{2}\left(M_{1}\right)+c^{2}\left(M_{2}\right) \bmod 2 .
$$

Next we introduce the concept of a boundary of a manifold. First, a curve $\gamma$ is a union of different 1-cells $c^{1}$. We allow $\gamma$ to be selfintersecting in the sense that any vertex may be contained in the closure of more than 21 -cells belonging to $\gamma$. We say that $\gamma$ is a curve without boundary and write $\partial \gamma=\phi$, if no vertex is contained in the closure of an odd number of different 1-cells belonging to $\gamma$.

For two curves $\gamma_{1}$ and $\gamma_{2}$ we define their symmetric sum as

$$
\gamma_{1}+\gamma_{2}=\gamma_{1} \cup \gamma_{2} \backslash \gamma_{1} \cap \gamma_{2} \text {. }
$$

For any manifold $M$ we define its boundary $\partial M$ to consist exactly of those 1-cells which are in the closure of an odd number of 2-cells belonging to $M$. With this definition we have

$$
\partial(\partial M)=\phi,
$$

and

$$
\partial\left(M_{1}+M_{2}\right)=\partial M_{1}+\partial M_{2},
$$

i.e. $\partial$ is a homomorphism of the group of all manifolds [with the group operation (4)] into the group of all curves without boundary [with the group operation (6)]. That the curves without boundaries form a group follows from a relation similar to (8) for curves

$$
\partial\left(\gamma_{1}+\gamma_{2}\right)=\partial \gamma_{1}+\partial \gamma_{2},
$$

where the sum on the right-hand side is now the symmetric sum for vertices.

To any $\gamma$ with $\partial \gamma=\phi$ we now associate the (possibly empty) sets

$$
C(\gamma)=\{M \mid \partial M=\gamma\},
$$

and

$$
C_{\Lambda}(\gamma)=\{M \in \Lambda, \operatorname{dist}(M, \partial \Lambda) \geqq a \mid \partial M=\gamma\} .
$$

Note that $C_{\Lambda}(\gamma)=\phi$ if $\gamma$ is not contained in $\Lambda \backslash \partial \Lambda$.

We remark that only local properties are involved in the definition of these ensembles. In particular, we made no restriction on the connectivity. This contrasts with many other models discussed so far (see e.g. [F] for a discussion on this point).

Since

$$
C\left(\gamma_{1}\right)+C\left(\gamma_{2}\right) \cong C\left(\gamma_{1}+\gamma_{2}\right),
$$

by (8), and similarly

$$
C_{\Lambda}\left(\gamma_{1}\right)+C_{A}\left(\gamma_{2}\right) \subseteq C_{\Lambda}\left(\gamma_{1}+\gamma_{2}\right),
$$

we have the following:

Lemma 1. $C(\phi)$ and $C_{A}(\phi)$ are subgroups of $\mathscr{G}$ and $\mathscr{G}_{A}$, respectively. Any nonempty $C(\gamma)$ and $C_{A}(\gamma)$ is an orbit under $C(\phi)$ and $C_{A}(\phi)$, respectively. $C(\phi)$ and $C_{A}(\phi)$ operate fixed point free on $C(\gamma)$ and $C_{A}(\gamma)$, respectively. 
Proof. The first statement is a trivial consequence of rels. (12) and (13) applied to the case $\gamma_{1}=\gamma_{2}=\phi$. By the same relations $C(\phi)$ and $C_{\Lambda}(\phi)$ operate as transformation groups on $C(\gamma)$ and $C_{A}(\gamma)$, respectively. As for the second statement, let $M_{1}, M_{2} \in C(\gamma)$. Then by (8) we have

$$
\partial\left(M_{1}+M_{2}\right)=\partial M_{1}+\partial M_{2}=\gamma+\gamma=\phi .
$$

Hence $M_{1}+M_{2}=N \in C(\phi)$. Adding $M_{2}$ on both sides gives $M_{1}=M_{2}+N$. Next assume $M_{1}=M_{1}+N$ for $M_{1} \in C(\gamma)$ and $N \in C(\phi)$. Adding $M_{1}$ on both sides gives $\phi=N$. The discussion for $C_{\Lambda}(\gamma)$ is similar.

For any finite manifold we define its area by

$$
\operatorname{Area}(M)=a^{2} \sum_{c^{2} \in M} 1=a^{2} \sum_{c^{2}} c^{2}(M) .
$$

We will introduce partition functions with this area as the action and $C_{A}(\gamma)(\gamma$ fixed) as the underlying statistical Gibbs ensemble. We will assume $\gamma$ to be finite and $C_{\Lambda}(\gamma)$ to be nonempty. Hence there exists a (not necessarily unique) minimal surface $M(\gamma)$ defined by the property

$$
\operatorname{Area}(M(\gamma))=\inf _{M \in C(\gamma)} \operatorname{Area}(M) .
$$

For $\gamma=\phi$ we set $M(\phi)=\phi$. Also from now on we will assume $\Lambda$ to be so large that $M(\gamma) \in C_{\Lambda}(\gamma)$. We may now perform a trivial additive renormalization on $C_{\Lambda}(\gamma)$ by setting

$$
E_{\gamma}(M)=\operatorname{Area}(M)-\operatorname{Area}(M(\gamma)) \geqq 0 .
$$

For $\beta>0$ the partition function $Z_{\Lambda}(\gamma, \beta)$ is now given as

$$
Z_{\Lambda}(\gamma, \beta)=\sum_{M \in C_{A}(\gamma)} \exp -\beta E_{\gamma}(M) \text {. }
$$

Note that $\beta$ has the dimension of an inverse area.

By Lemma 1 we may rewrite the partition function as

$$
Z_{\Lambda}(\gamma, \beta)=\sum_{N \in C_{\Lambda}(\phi)} \exp -\beta E_{\gamma}(M(\gamma)+N) .
$$

From (4) we deduce

$$
\operatorname{Area}(N)-\operatorname{Area}(M(\gamma)) \leqq \operatorname{Area}(M(\gamma)+N) \leqq \operatorname{Area}(N)+\operatorname{Area}(M(\gamma)) \text {. }
$$

Setting

$$
z_{\Lambda}(\gamma, \beta)=\frac{Z_{\Lambda}(\gamma, \beta)}{Z_{\Lambda}(\phi, \beta)},
$$

this gives our first important estimate

$$
1 \leqq z_{\Lambda}(\gamma, \beta) \leqq e^{2 \beta \operatorname{Area}(M(\gamma))},
$$

valid uniformly in $\Lambda$. This sharpens a result obtained in [Sch] by much more elaborate methods.

In a next step, we will rewrite $z_{A}(\gamma, \beta)$ as a quantity entirely computable in the statistical ensemble $C_{A}(\phi)$. Let \langle\rangle$_{A, \gamma}(\beta)$ denote the expectations in the Gibbs ensemble $C_{\Lambda}(\gamma)$ at inverse temperature $\beta$. 
Let $M_{1}, M_{2} \in C_{A}(\gamma)$ be arbitrary. Then by Lemma 1 we have

$$
\sum_{N \in C_{\Lambda}(\phi)} e^{-\beta \operatorname{Area}\left(M_{1}+N\right)}=\sum_{N \in C_{\Lambda}(\phi)} e^{-\beta \operatorname{Area}\left(M_{2}+N\right)} .
$$

Now, considered as a function of $N$

$$
\begin{aligned}
\operatorname{Area}(M+N) & =\operatorname{Area}(M)+\operatorname{Area}(N)-2 \operatorname{Area}(M \cap N) \\
& =\operatorname{Area}(M)+\operatorname{Area}(N)-2 a^{2} \sum_{c^{2} \in M} c^{2}(N) .
\end{aligned}
$$

Inserting this into (22) gives us the following important identities

$$
\begin{aligned}
e^{-\beta \operatorname{Area}\left(M_{1}\right)}\left\langle e^{2 \beta a^{2} \underset{c^{2} \in M_{1}}{\Sigma} c^{2}}\right\rangle_{\Lambda, \phi}(\beta) & =e^{-\beta \operatorname{Area}\left(M_{1}\right)}\left\langle e^{2 \beta a^{2}{ }_{c^{2} \in M_{2}}^{\Sigma} c^{2}}\right\rangle_{\Lambda, \phi}(\beta) \\
& =e^{-\beta \operatorname{Area}(M(\gamma))} z_{\Lambda}(\gamma, \beta),
\end{aligned}
$$

provided only that $\partial M_{1}=\partial M_{2}=\gamma$. The first part of rel. (24) may be viewed as a Stokes theorem for our model of random surfaces.

As a special application we obtain

Lemma 2. The quantity $z_{\Lambda}(\gamma, \beta)$ is a Green's function in the Gibbs ensemble $C_{\Lambda}(\phi)$ :

$$
z_{\Lambda}(\gamma, \beta)=\left\langle e^{2 \beta a^{2}{ }_{c^{2} \in M(\gamma)}^{\Sigma} c^{2}}\right\rangle_{\Lambda, \phi}(\beta) .
$$

Note that

$$
e^{2 \beta a^{2} c^{2}}=1+\left(e^{2 \beta a^{2}}-1\right) c^{2},
$$

so the right-hand side of (25) is an expectation of a polynomial with positive coefficients in the dynamical variables $c^{2}$ of order Area $(M(\gamma)) / a^{2}$.

In view of Lemma 2, rel. (24) now has the following important interpretation. For any $M \in C_{\Lambda}(\gamma)$ with $\partial M=\gamma$ and given $\beta>0$, we may view the observable

$$
N \rightarrow e^{-\beta \operatorname{Area}(M)} e^{2 \beta a^{2}{ }_{c^{2} \in M}^{\Sigma} c^{2}(N)}
$$

on $C_{A}(\phi)$ as a creation operator for the boundary $\gamma$ in the Gibbs ensemble $C_{A}(\phi)$. In fact, by (24) the expectation value of this observable is independent of the particular choice of $M$.

As an immediate consequence of Lemma 2 we have

Lemma 3. $z_{\Lambda}(\gamma, \beta)$ is monotonically increasing in $\gamma$ in the sense that

$$
z_{\Lambda}\left(\gamma^{\prime}, \beta\right) \geqq z_{\Lambda}(\gamma, \beta), \text { provided } M\left(\gamma^{\prime}\right) \supseteqq M(\gamma) .
$$

In the same fashion, we may express expectations with respect to \langle\rangle$_{A, \gamma}(\beta)$ by quotients of expectations with respect to \langle\rangle$_{A, \phi}(\beta)$ :

Lemma 4. For any polynomial $P$ in the basic dynamic variables $c^{2}$,

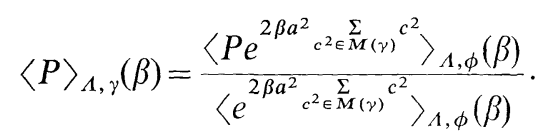

Now in $[\mathrm{Sch}]$ we proved the existence of the bulk limit for the free energy per unit volume for a large class of interactions on $C(\phi)$, including the action given by 
the area. The same was shown to hold for expectations. Actually, the proof was given for $d=3$ only, but it extends easily to arbitrary $d$. We collect this in

Lemma 5. For all $\beta \geqq 0$, the bulk limits $z(\gamma, \beta)$ of $z_{\Lambda}(\gamma, \beta)$ and $\langle P\rangle_{\gamma}(\beta)$ of $\langle P\rangle_{\Lambda, \gamma}(\beta)$ exist, when $\Lambda$ tends to infinity in the sense of van Hove.

By Lemmas 2 and 4 all interesting quantities may be obtained as (quotients of) expectation values in the ensemble $C_{\Lambda}(\phi)$. We will see in Sect. 4 that in particular $z_{\Lambda}(\gamma, \beta)$ will provide us with interesting information. Hence in a Monte Carlo computer simulation it will be sufficient to simulate the Gibbs distribution on $C_{\Lambda}(\phi)$.

Now a fast simulation for this is well known [SG]. It has also been used in [Sch] for the case $d=3$, where, however, still separate simulations for $C_{\Lambda}(\gamma)$ and $C_{\Lambda}(\phi)$ were performed. This simulation for $C_{A}(\phi)$ is briefly described as follows. At $t=0$ one starts with the empty manifold. At each computer time a manifold $M(t)$ is stored. The upgrading at $t^{\prime}=t+1$ is performed by a local surgery in forming the trial configuration

$$
M_{\text {trial }}=M(t)+\partial\left(c^{3}\right)
$$

for a fixed 3-cell $c^{3}$. If $M(t)$ has empty boundary so has $M_{\text {trial }} \cdot M_{\text {trial }}$ is then accepted as $M(t+1)$ with the usual acceptance rate derived from the Gibbs factor. Sweeping through all 3-cells $c^{3}$ gives the desired upgrading.

Ergodicity, which ensures that the Gibbs distribution is the unique equilibrium distribution under the upgrading (30), is now equivalent to the fact that the group $C_{\Lambda}(\phi)$ is generated by the elements of the form $\partial c^{3}$. We state this as a separate

Lemma 6. If $\Lambda$ is a rectangular box, then the group $C_{\Lambda}(\phi)$ is generated by the $\partial c^{3}\left(\operatorname{dist}\left(c^{3}, \partial \Lambda\right) \leqq 1\right)$. In particular, the upgrading defined by (30) is ergodic.

The proof is given in the appendix.

To obtain a further control on the $\gamma$-dependence of $z_{\Lambda}(\gamma, \beta)$, we will prove the physical positivity of the expectation \langle\rangle$_{\Lambda, \phi}(\beta)$. Let $\Lambda$ be a rectangular box and $H$ a half plane parallel to one of the sides of $\Lambda$, dividing $\Lambda$ into two closed sets $\Lambda_{+}$and $\Lambda_{-}$, equal under reflection. Let $\vartheta=\vartheta_{H}$ denote the reflection with respect to this plane. Let $\mathscr{P}\left(\Lambda_{ \pm}\right)$denote the algebra over $\mathbb{C}$ generated by the dynamical variables $c^{2}$ of those 2-cells intersecting $\Lambda_{+}$and $\Lambda_{-}$, respectively. Note that $\mathscr{P}\left(\Lambda_{+}\right) \cap \mathscr{P}\left(\Lambda_{-}\right)$is nonempty. Let $\Theta$ denote the antilinear algebra automorphism $\Theta: \mathscr{P}\left(\Lambda_{ \pm}\right) \rightarrow \mathscr{P}\left(\Lambda_{\mp}\right)$ induced by $\vartheta$.

Lemma 7. For any $f \in \mathscr{P}\left(\Lambda_{+}\right)$,

$$
\langle f \Theta f\rangle_{\Lambda, \phi}(\beta) \geqq 0 .
$$

Proof. By our previous remarks, we may identify $C_{\Lambda}(\phi)$ with a subgroup of $\mathscr{G}_{\Lambda}$. For any function $F$ on $\mathscr{G}_{A}$, let

$$
(F)_{\Lambda}(\beta)=\sum_{g \in \mathscr{C}_{\Lambda}}\left(F(g) e^{-\beta \sum_{c^{2}} c^{2}}\right) \quad\left(g=\left\{c^{2}\right\}\right) .
$$

The restriction to the subensemble $C_{\Lambda}(\phi)$ is now obtained by introducing a suitable projection operator. First, let

$$
P_{\partial \Lambda}=\prod_{c^{2}: \operatorname{dist}\left(c^{2}, \partial \Lambda\right)=0}\left(1-c^{2}\right)
$$


be the projection operator enforcing the boundary condition in (11). From $P_{\partial \Lambda}=P_{\partial \Lambda}^{2}$ and $\Theta P_{\partial \Lambda}=P_{\partial \Lambda}$, we have $P_{\partial \Lambda}=P_{\partial \Lambda} \Theta P_{\partial \Lambda}$. Next for a given 1-cell $c^{1}$, let $S_{c^{1}}^{j}(1 \leqq j \leqq 2(d-1))$ be the $j^{\text {th }}$ elementary symmetric polynomial in the $2(d-1)$ 2-cells $c^{2}$ which contain $c^{1}$ and their closure. Then it is easily seen that there are real $a_{j}$ such that $\left(S_{c^{1}}^{0}=1\right)$

$$
Q_{c^{1}}=\sum_{j=0}^{2(d-1)} a_{j} S_{c^{1}}^{j}
$$

is zero exactly when an odd number of these $c^{2}$ take the value 1 and one otherwise. For example, for $d=3$ this polynomial takes the explicit form

$$
Q_{c^{1}}=1-S_{c^{1}}^{1}+2 S_{c^{1}}^{2}-4 S_{c^{1}}^{3}+8 S_{c^{1}}^{4} .
$$

In particular, $Q_{c^{1}}=\left(Q_{c^{1}}\right)^{2}$, and since $\Theta S_{c^{1}}^{j}=S_{\vartheta c^{1}}^{j}$ for all $j$, we have $\Theta Q_{c^{1}}=Q_{\vartheta c^{1}}$. Thus the product $Q=\prod_{c^{1}} Q_{c^{1}}$ satisfies $Q^{2}=Q, \Theta Q=Q$. Now let

$$
P=P_{\partial \Lambda} Q,
$$

such that

Using (32), we also have

$$
P=P \Theta P \text {. }
$$

$$
\langle f \Theta f\rangle_{A, \phi}(\beta)=Z_{\Lambda}(\phi, \beta)(f \Theta f P)_{\Lambda}(\beta)=Z_{\Lambda}(\phi, \beta)(P f \Theta(P f))_{\Lambda}(\beta) .
$$

Now the right-hand side of (37) is trivially seen to be $\geqq 0$ and Lemma 7 is proved.

To exploit this result, note that $\vartheta$ induces a map $\gamma \rightarrow \vartheta \gamma$ on the set of boundaries. A direct consequence of Lemmas 2 and 6 is

Lemma 8. Let the boundaries $\gamma_{1}$ and $\gamma_{2}$ be such that $M\left(\gamma_{1}\right)$ and $M\left(\gamma_{2}\right)$ live in $\Lambda_{+}$. Then for all $\beta>0$,

$$
z_{\Lambda}\left(\gamma_{1}+\vartheta \gamma_{2}, \beta\right) \leqq z_{\Lambda}\left(\gamma_{1}+\vartheta \gamma_{1}, \beta\right)^{1 / 2} z_{\Lambda}\left(\gamma_{2}+\vartheta \gamma_{2}, \beta\right)^{1 / 2} .
$$

This proof of reflection positivity was essentially based on the fact that our manifolds were defined by a local condition. By similar arguments therefore reflection positivity in the above form continues to hold for the ensemble of selfavoiding manifolds as discussed for example in [KT, Sch]. For these ensembles we do not know of any analogue of Lemma 2.

\section{Equivalence to the Ising Model $(d+3)$ and $Z_{2}$ Gauge Theory}

In this section we recall the well known isomorphy to the $d=3$ Ising model, by which elements in $C(\phi)$ correspond to Peierls interfaces. More precisely the correspondence is obtained by assigning a dynamical variable to each 3-cell $c^{3}$, also denoted by $c^{3}$ and taking the values \pm 1 (we deviate here from the standard notation which employs the symbol $\sigma$ ). We impose the boundary conditions

$$
c^{3}=1, \quad \operatorname{dist}\left(c^{3}, \partial \Lambda\right)=0 .
$$

Then the relation between the dynamical 2-cell variables of our random surface model and the 3-cell variables of the Ising model is given as

$$
c^{2}=\frac{1}{2}\left(1-c^{3} c^{3}\right),
$$


where $c^{3}$ and $c^{3 \prime}$ are the two unique 3-cells containing $c^{2}$ in the intersection of their closures. In words, $c^{2}$ belongs to an interface iff $c^{3}$ and $c^{3 \prime}$ have opposite sign. Furthermore, by (40) the actions of the two theories agree modulo an additive constant provided,

$$
\beta a^{2}=2 \beta_{\text {Ising }}
$$

so we have

$$
\langle\cdot\rangle_{\Lambda, \phi}(\beta)=\langle\cdot\rangle_{\Lambda}\left(\beta_{\text {Ising }}\right),
$$

where the right-hand side denotes the expectation values for the Ising model with boundary conditions (39). Also (40) is the translation code for the basic dynamical observables.

This correspondence gives a new proof of the bulk limit when $d=3$. Moreover, we have

Lemma 9. For $\beta a^{2}<2 \beta_{\text {Ising }}$ (critical) and any $\gamma$, the expectations $\langle\cdot\rangle_{\gamma}(\beta)$ exhibit exponential clustering with a mass gap bounded below by the corresponding mass gap of the Ising model.

Remark. This results indicates that the critical value $\beta_{0}$ for our random surface model should satisfy $\beta_{0} a^{2}>2 \beta_{\text {Ising }}$ (critical). This also suggested by the numerical results in [Sch].

To conclude this section, we translate the first part of relation (24) into the corresponding Ising model relation. It may be viewed as an Ising model version of Stokes theorem and is well known [KC].

Lemma 10. In $d=3$, let $M_{1}$ and $M_{2}$ be any union of 2-cells such that $M_{1}+M_{2}$ is a Peierls interface. Then

$$
\left\langle\exp -2 \beta_{\text {Ising }} \Sigma_{1} c^{3} c^{3 \prime}\right\rangle_{\Lambda}\left(\beta_{\text {Ising }}\right)=\left\langle\exp -2 \beta_{\text {Ising }} \Sigma_{2} c^{3} c^{3 \prime}\right\rangle_{\Lambda}\left(\beta_{\text {Ising }}\right),
$$

where the sum $\Sigma_{j}$ extends over all pairs of 3-cells $c^{3}, c^{3 \prime}$ such that the intersection of their closure contains a 2-cell in $M_{j}$.

We recall the direct proof [KC]: For the given Peierls interface $M_{1}+M_{2}$, let $T$ be the union of all 3-cells for which $c^{3}$ would take the value -1 , given the boundary condition (39). Then (43) follows by making the variable substitution $c^{3} \rightarrow-c^{3}$ for all 3-cells in $T$.

It is now obvious how relation (43) extends to arbitrary $d$. The crucial property again is that the Peierls interfaces form a group under the symmetric sum.

Next we recall the well known equivalence to the $Z_{2}$ lattice gauge theory (see e.g. $[\mathrm{BDI}])$. Let $U_{c^{1}}= \pm 1$ be the lattice bond variable and

$$
U_{c^{2}}=\prod_{c^{1} \in \partial c^{2}} U_{c^{1}} \quad(= \pm 1)
$$

the corresponding plaquette variable. The partition function at inverse temperature $\beta_{g}$ takes the form

$$
\begin{aligned}
Z_{\Lambda}\left(\beta_{g}\right) & =\sum_{U_{c^{1}}= \pm 1} \exp \beta_{g} \sum_{c^{2}}\left(U_{c^{2}}-1\right) \\
& \left.=\left(\exp -\beta_{g} \cosh \beta_{g}\right)^{\#\left(c^{2} \in \Lambda\right.}\right) \sum_{U_{c^{1}}= \pm 1} \prod_{c^{2}}\left(1+U_{c^{2}} \tanh \beta_{g}\right) .
\end{aligned}
$$


Expanding the product and doing the sum, only nonvanishing contributions arise when an even number of plaquettes meet at any bond. This gives

$$
\left.Z_{\Lambda}\left(\beta_{g}\right)=\left(\exp -\beta_{g} \cosh \beta_{g}\right)^{\#\left(c^{2} \in \Lambda\right)} 2^{\#\left(c^{1} \in \Lambda\right.}\right) Z_{\Lambda}(\gamma=\phi, \beta),
$$

where $\beta$ and $\beta_{g}$ are related by the duality transformation

$$
\tanh \beta_{g}=\exp -\beta \text {. }
$$

Similarly we obtain for the unrenormalized Wilson loop expectation

$$
\sum_{U_{c}= \pm 1} \prod_{c^{1} \in \gamma} U_{c^{1}} \exp \beta_{g}\left(\sum_{c^{2}} U_{c^{2}}-1\right)=\left(\cosh \beta_{g} \exp -\beta_{g}\right)^{\#\left(c^{2} \in \Lambda\right)} 2^{\#\left(c^{1} \in \Lambda\right)} Z_{\Lambda}(\gamma, \beta) .
$$

Therefore with the duality relation (47) our $Z_{\Lambda}(\gamma, \beta)$ is just the Wilson loop expectation, giving an alternative proof of reflection positivity and Stokes theorem in the form of relation (24).

\section{String Tension and Glueball Mass}

In $[\mathrm{Sch}]$ we interpreted the quantity

$$
F(\gamma, \beta)=\ln z(\gamma, \beta)=\lim _{\Lambda \rightarrow \infty} \ln z_{\Lambda}(\gamma, \beta)=\lim _{\Lambda \rightarrow \infty} \ln \left(Z_{\Lambda}(\gamma, \beta)-Z_{\Lambda}(\phi, \beta)\right)
$$

as the polarization energy of the boundary $\gamma$. By (21) it obeys the estimate

$$
0 \leqq F(\gamma, \beta) \leqq 2 \beta \text { Area }(M(\gamma)) \text {. }
$$

In fact, in terms of the Nambu-Goto $[\mathrm{N}, \mathrm{G}]$ string quantization picture, the connected component of $M(\gamma)+N(N \in C(\phi))$ containing $\gamma$ may be interpreted as the string associated to $\gamma$. The presence of the other components of $M(\gamma)+N$, which therefore have empty boundary, are interpreted as vacuum polarization effects due to glueballs (I am indebted to G. Sterman for an enlightening discussion on this point). Formally therefore, as in the Gell-Man Low formula, $z_{A}(\phi, \beta)$ serves to "factor out" these vacuum polarization effects. Considering $F(\gamma, \beta)$ as a basic quantity therefore replaces the connectivity condition placed on a random surface in other models (see e.g. [F]).

Now consider the dimensionless quantity

$$
f(\beta, a)=\lim _{\gamma: \operatorname{Area}(M(\gamma)) \rightarrow \infty} \frac{a^{2} F(\gamma, \beta)}{\operatorname{Area}(M(\gamma))},
$$

provided the limit exists. Here we have indicated the dependence on the lattice size $a$. By the equivalence to the $Z_{2}$ lattice gauge model, the intrinsically defined quantity

$$
\alpha(\beta, a)=f(\beta, a)-\beta a^{2}
$$

may serve as a definition of the string tension. The term $\beta a^{2}$ has been subtracted in order to undo the additive renormalization performed in (17). For a definition in the same spirit but in different models, see again e.g. $[\mathrm{F}]$ and the references quoted 
there. By (45) we have

$$
-\beta a^{2} \leqq \alpha(\beta, a) \leqq \beta a^{2}
$$

With this definition, the string tension in this model as well as for the ensemble of selfavoiding manifolds seems to have the desired properties. Indeed by (53) we have

$$
\alpha(0, a)=0
$$

Also in [Sch] we performed a computer simulation to evaluate $\frac{d}{d \beta} \alpha(\beta, a=1)$ (see, in particular, Fig. 3 in $[\mathrm{Sch}])$. The results obtained there indicate the existence of a $\beta_{0}$ with

Also

$$
\frac{d}{d \beta} \alpha(\beta, a=1)=0, \quad 0 \leqq \beta<\beta_{0} .
$$

$$
\left|\frac{d}{d \beta} \alpha(\beta, a=1)\right|<\text { const for all } \beta,
$$

whereas the second derivative is discontinuous at $\beta_{0}$. Granted these results, the string tension vanishes for $\beta<\beta_{0}$. Also if $\alpha(\beta, a=1)$ for $\beta>\beta_{0}$ is assumed to be of the form $\operatorname{const}\left(\beta-\beta_{0}\right)^{\mu}$, then we have

$$
1<\mu<2 \text {. }
$$

Since the related model of selfavoiding random surfaces shares similar features (with a shifted critical value for $\beta$ ), we would not be surprised if these two models belong to the same universality class.

We turn to a definition of the glueball mass. Let $\gamma_{0}(x)$ denote a translate of the loop $\gamma_{0}$ by $x \in(a \mathbb{Z})^{d}$. If the quantity

$$
g\left(\gamma_{0}, \beta, x\right)=\ln \left(z\left(\gamma_{0}+\gamma_{0}(x), \beta\right) z^{-2}\left(\gamma_{0}, \beta\right)\right)
$$

has an exponential decay rate as $x \rightarrow \infty$, we may use this as the definition of the glueball mass for the loop $\gamma_{0}$. Indeed, for large $|x|$ we have

$$
M\left(\gamma_{0}+\gamma_{0}(x)\right)=M\left(\gamma_{0}\right)+M\left(\gamma_{0}\right)(x)
$$

where $M(x)$ denotes the translate of $M$ by $x$. We expect cluster properties (at least for small $\beta$ ) of \langle\rangle$_{\phi}(\beta)$, the bulk limit of \langle\rangle$_{\Lambda, \phi}(\beta)$. Applying Lemma 2 therefore should give

$$
\lim _{|x| \rightarrow \infty} z\left(\gamma_{0}+\gamma_{0}(x), \beta\right)=z\left(\gamma_{0}, \beta\right)^{2} .
$$

We turn to an analytic discussion of these quantities. First, as a consequence of Lemma 7, we have

$$
F\left(\gamma_{1}+\vartheta \gamma_{2}, \beta\right) \leqq \frac{1}{2} F\left(\gamma_{1}+\vartheta \gamma_{1}, \beta\right)+\frac{1}{2} F\left(\gamma_{2}+\vartheta \gamma_{2}, \beta\right)
$$

for any reflection with respect to a plane not cutting or separating $M\left(\gamma_{1}\right)$ and $M\left(\gamma_{2}\right)$. Let now $\gamma(L, M)$ denote the boundary of a rectangle of sidelengths $a L$ and $a M\left(L, M \in \mathbb{Z}^{+}\right)$. Then (61) gives

$$
F\left(\gamma\left(L+L^{\prime}, M\right), \beta\right) \leqq \frac{1}{2} F(\gamma(2 L, M), \beta)+\frac{1}{2} F\left(\gamma\left(2 L^{\prime}, M\right), \beta\right),
$$


and similarly for $L$ and $M$ interchanged. This gives the

Theorem 11. For all $\beta \geqq 0$, the string tension exists when the limit $\gamma \rightarrow \infty$ is taken over the sequence $\gamma(L, M)$ with $L \rightarrow \infty$ and $M \rightarrow \infty$.

Next we turn to discussion of the quantity $g\left(\gamma_{0}, \beta, x\right)$. For simplicity we will take $\gamma_{0}$ to lie in a plane and $x$ to be a translation orthogonal to that plane, i.e. $x=|x| \mathbf{e}$, where $\mathbf{e}$ is orthogonal to the plane spanned by $\gamma_{0}$. Set

$$
H(|x|)=F\left(\gamma_{0}+\gamma_{0}(x), \beta\right) .
$$

Applying Lemma 7 again gives

$$
H(|x|+|y|) \leqq \frac{1}{2} H(2|x|)+\frac{1}{2} H(2|y|),
$$

i.e. $H(r)$ is convex in $r \in a\left(\mathbb{Z}^{+}+1\right)$. Since $H(r)$ is bounded, see (50), this is only possible if $H(|x|)$ is monotonically nonincreasing in $|x|$. Thus we have proved

Lemma 12. $g\left(\gamma_{0}, \beta, x\right)$ is monotonically decreasing in $|x|$ for $\gamma_{0}$ lying in a plane and $x$ being translation orthogonal to that plane.

By our previous discussion we expect $g\left(\gamma_{0}, \beta, x\right)$ to tend to zero as $|x| \rightarrow \infty$. Assuming this to be the case, the glueball mass for the loop $\gamma_{0}$ is defined by

$$
\begin{aligned}
m\left(\gamma_{0}, \beta, a\right) & =-\lim _{|x| \rightarrow \infty} \frac{1}{|x|} \ln \left(\left|\ln \frac{z\left(\gamma_{0}+\gamma_{0}(x), \beta\right)}{z\left(\gamma_{0}, \beta\right)^{2}}\right|\right) \\
& =-\lim _{|x| \rightarrow \infty} \frac{1}{|x|} \ln \left(\left|F\left(\gamma_{0}+\gamma_{0}(x), \beta\right)-2 F\left(\gamma_{0}, \beta\right)\right|\right) .
\end{aligned}
$$

In fact, by Lemma 9 we have the

Theorem 13. For $d=3$ and with $\beta a^{2}=2 \beta_{\text {Ising }}<2 \beta_{\text {Ising }}$ (critical) the glueball mass $m\left(\gamma_{0}, \beta, a\right)$ is bounded below by the mass gap $m\left(\beta_{\text {Ising }}\right)$ of the Ising model.

To conclude this section, we want to use $z\left(\gamma_{0}+\gamma_{0}(x), \beta\right)$ to prove the following Theorem 14. In the thermodynamic limit the translation invariant quantity

satisfies the estimate

$$
\left\langle c^{2}\right\rangle_{\phi}(\beta)=\lim _{\Lambda \rightarrow \infty}\left\langle c^{2}\right\rangle_{\Lambda, \phi}(\beta)
$$

for all $\beta \geqq 0$.

$$
0 \leqq\left\langle c^{2}\right\rangle_{\phi}(\beta) \leqq \frac{1}{2}
$$

In words it says that the (translation invariant) probability for a plaquette to belong to a manifold without boundary [i.e. to an element of $C(\phi)]$ is less than $1 / 2$. Although we consider this result to be nontrivial (except for $d=3$, see Sect. 3), it is not surprising. Indeed, since each 1-cell has to be contained in the closure of an even number of 2-cells of the manifold, we expect this condition to prevent a 2-cell to belong to an element of $C(\phi)$ at least half of the times.

Proof. Let $\gamma_{0}$ be the boundary of an elementary 2-cell and let again $x$ be a translation orthogonal to this 2-cell. Also let $M_{x}$ be the sides of the cylinder formed 
by $\gamma_{0}$ and $\gamma_{0}(x)$, such that Area $\left(M_{x}\right)=4 a|x|$. Then by (24),

$$
z\left(\gamma_{0}+\gamma_{0}(x), \beta\right)=e^{2 \beta a^{2}-4 \beta a|x|}\left\langle e^{2 \beta a^{2}{ }_{c^{2} \in M_{x}}^{\Sigma} c^{2}}\right\rangle_{\phi}(\beta) .
$$

Applying Jensen's inequality (see e.g. [Si]) we obtain

$$
\begin{aligned}
z\left(\gamma_{0}+\gamma_{0}(x), \beta\right) & \geqq e^{2 \beta a^{2}-4 \beta a|x|+2 \beta a^{2}{ }_{c^{2} \in M_{x}}^{\Sigma}\left\langle c^{2}\right\rangle_{\phi}}(\beta) \\
& =e^{2 \beta a^{2}-4 \beta a|x|+8 \beta a|x|\left\langle c^{2}\right\rangle_{\phi}}(\beta),
\end{aligned}
$$

where the equality follows from translation invariance. On the other hand, $z\left(\gamma_{0}+\gamma_{0}(x), \beta\right)$ is bounded in $|x|$. This is only possible if $(67)$ holds.

\section{Selfoverlapping Manifolds}

Up till now, the manifolds $M$ we considered were not allowed to fold onto themselves, i.e. each 2-cell $c^{2}$ appeared at most once in manifold. We will now allow a 2-cell $c^{2}$ to appear with multiplicity $c^{2}(M)$ between 0 and $n-1$ ( $n$ even). This corresponds to replacing $\mathbb{Z}_{2}$ in (1) and (2) by $\mathbb{Z}_{n}$. The set of manifolds again forms an abelian group with the group operation being given implicitly by

$$
c^{2}\left(M_{1}+M_{2} \equiv c^{2}\left(M_{1}\right)+c^{2}\left(M_{2}\right) \bmod n .\right.
$$

For given $M$ we define its boundary $\partial M$ to be the union of 1-cells $c^{1}$ for which

$$
\sum_{c^{2}: c^{1} \in \partial c^{2}} c^{2}(M) \equiv 1 \bmod 2 .
$$

Let now $C_{\Lambda}(\gamma, n)$ be the set of such selfoverlapping manifolds with boundary $\gamma$ and distance $\geqq a$ to $\partial \Lambda$. Because $n$ is even, rel. (13) and therefore Lemma 1 again hold. In analogy the partition function is now defined as

$$
Z_{\Lambda}(\gamma, \beta, \mu)=e^{\beta \operatorname{Area}(M(\gamma))} \sum_{M \in C_{\Lambda}(\gamma, n)} e^{-\beta a^{2} \sum_{c^{2}} c^{2}(M)}
$$

where we now consider our previous minimal surface $M(\gamma)$ as an element of $C_{\Lambda}(\gamma, n)$. To reexpress

$$
z_{\Lambda}(\gamma, \beta, n)=\frac{Z_{\Lambda}(\gamma, \beta, n)}{Z_{\Lambda}(\phi, \beta, n)},
$$

as an expectation in the ensemble $C_{\Lambda}(\phi, n)$, we need a formula which replaces (23). In fact, such a formula is given by

$$
c^{2}(M(\gamma)+N)=c^{2}(M(\gamma))+c^{2}(N)-n c^{2}(M(\gamma)) \prod_{i=0}^{n-2}\left(\frac{c^{2}(N)-i}{n-1-i}\right),
$$

where we have made use of the fact that $c^{2}(M(\gamma))$ is either zero or one. This gives

$$
z_{\Lambda}(\gamma, \beta, n)=\left\langle\exp \left(+\beta a^{2} n \sum_{c^{2} \in M(\gamma)} \prod_{i=0}^{n-2} \frac{\left(c^{2}-i\right)}{(n-1-i)}\right)\right\rangle_{\Lambda, \phi}(\beta, n),
$$

where \langle\rangle$_{\Lambda, \phi}(\beta, n)$ denotes the expectation in the ensemble $C_{\Lambda}(\phi, n)$ at inverse temperature $\beta$. Note that for $n=2(74)$ and (75) reduce to (23) and (25), respectively. 
Also Lemma 3 extends to the present case as does Stokes theorem [first part of rel. (24)].

Furthermore, physical positivity holds, since it follows by an easy adaption of the proof of Lemma 7. Since the thermodynamic limit can be established along the lines of [Sch], the string tension and the glueball mass are again well defined. It would be interesting to study the behaviour as $n \rightarrow \infty$, the case when no conditions are imposed on the number of times the manifold can fold onto itself.

Finally, the upgrading procedure has to be modified as follows. In addition to the local surgery (30) in an alternate step, one also adds the manifolds which are the double of one 2-cell. Running through all 2-cells in this way gives an ergodic upgrading.

\section{Coloured Strings}

In this section we want to propose a way to add colour to our surfaces. In the language of string quantization consider an (infinitely thin) string, hypothetically at rest and containing a Yang-Mills field line starting at a quark at one endpoint and ending in an antiquark at the other endpoint. This is simply the colour flux tube interpretation of the string. A covariant formulation for moving strings might therefore look as follows. Let $M$ be a 2-dimensional compact manifold (not necessarily connected) isometrically embedded in some $\mathbb{R}^{d}$ and with boundary $\partial M=\gamma$. The action considered so far was $\operatorname{Vol}(M)$. Let in addition a Yang-Mills field be given on $M$ with a curvature 2-form $F$ (with values in the Lie algebra of some compact semisimple Lie group $G$ ). Then we might look at the action

$$
\int_{M} d \mathrm{vol}+g_{0}^{-2} \int_{M}\|F\|^{2} d \mathrm{vol},
$$

possibly with some boundary conditions on $\gamma$ for the Yang-Mills potential. $g_{0}$ is a coupling constant and the second term is, of course, the usual Yang-Mills action (the norm being obtained from the Killing form and the metric on $M$ ). Thus the action (76) is a functional of $M$ and the Yang-Mills potential on $M$.

To obtain a lattice version for this action, we simply combine the discussion in Sect. 2 with the usual Wilson lattice gauge formulation [W] as follows. There a lattice gauge configuration in a box $\Lambda$ is given by a family of group elements

$$
\left\{g_{c^{1}}\right\}_{c^{1} \in \Lambda}, \quad g_{c^{1}} \in G .
$$

Here the 1-cells (bonds) are assumed to be oriented and as usual we set

$$
g_{-c^{1}}=\left(g_{c^{1}}\right)^{-1} .
$$

The elements (77) form a group. Let

$$
d \mu_{\Lambda}\left(\left\{g_{c^{1}}\right\}\right)=\prod_{c^{1} \in \Lambda} d \mu\left(g_{c^{1}}\right)
$$

be its normalized Haar measure, where $d \mu(\cdot)$ is the normalized Haar measure on $G$. For a given orientation of $c^{2}$, let

$$
g_{\partial c^{2}}=\prod_{c^{1} \in \partial c^{2}} g_{c^{1}}
$$


be the associated plaquette group element, unique up to conjugation. Let $\chi$ be any real character of $G$ belonging to a locally faithful representation and normalized by $\chi(1)=1$. Then by Schwarz inequality

$$
|\chi(g)| \leqq 1 \text { for } g \in G .
$$

For $\beta, g_{0}>0$ we now define partition functions

$$
\begin{aligned}
Z_{\Lambda}\left(\gamma, \beta, g_{0}\right)= & \exp \beta \operatorname{Area} M(\gamma) \sum_{M \in C_{A}(\gamma)} \int d \mu_{\Lambda}\left(\left\{g_{c^{1}}\right\}\right) \\
& \cdot \exp -\sum_{c^{2} \in \Lambda} \beta\left(1+g_{0}^{-2}\left(1-\chi\left(g_{\partial c^{2}}\right)\right) c^{2}(M) .\right.
\end{aligned}
$$

Note that for a given $M$ in this sum, all those $g_{c^{1}}$ integrate out trivially, for which $c^{1}$ does not belong to the closure of a plaquette $c^{2}$ lying in $M$, so that indeed the YangMills field lives on $M$. Let \langle\rangle$_{A}\left(\gamma, \beta, g_{0}\right)$ denote the corresponding expectations. Using again rel. (23), for the quantity

we now obtain the identity

$$
z_{\Lambda}\left(\gamma, \beta, g_{0}\right)=\frac{Z_{\Lambda}\left(\gamma, \beta, g_{0}\right)}{Z_{\Lambda}\left(\phi, \beta, g_{0}\right)},
$$

$$
z_{\Lambda}\left(\gamma, \beta, g_{0}\right)=\left\langle\exp \sum_{c^{2} \in M(\gamma)} \beta\left(2 c^{2}+g_{0}^{-2}\left(1-\chi\left(g_{\partial c^{2}}\right)\left(2 c^{2}-1\right)\right)\right\rangle_{A}\left(\phi, \beta, g_{0}\right) .\right.
$$

This identity leads to the following estimates. Set

Then [see (25)]

$$
\chi_{-}=\inf _{g \in G} \chi(g) \geqq-1 .
$$

$$
\begin{aligned}
& z_{\Lambda}(\gamma, \beta) \exp -\beta g_{0}^{-2}\left(1-\chi_{-}\right) \text {Area } M(\gamma) \\
& \quad \leqq z_{\Lambda}\left(\gamma, \beta, g_{0}\right) \leqq z_{\Lambda}(\gamma, \beta) \exp \beta g_{0}^{-2}\left(1-\chi_{-}\right) \text {Area } M(\gamma) .
\end{aligned}
$$

Combining the physical positivity established in Sect. 2 for the random surfaces with the physical positivity for lattice gauge theories [OSe] (see also e.g. [S]), we obtain physical positivity for our combined model. In particular, we may now define the string tension $\alpha\left(\beta, g_{0}\right)$ and the glueball mass in analogy to Sect. 4 . Relation (86) leads to the following estimate for the string tension.

$$
-\beta g_{0}^{-2}\left(1-\chi_{-}\right)+\alpha(\beta) \leqq \alpha\left(\beta, g_{0}\right) \leqq \beta g_{0}^{-2}\left(1-\chi_{-}\right)+\alpha(\beta) .
$$

As for a Monte Carlo simulation the present approach, however, does not seem to offer any advantage for calculating the string tension or the glueball mass vis a vis the usual Wilson loop approach in lattice gauge theories. A comparison on the other hand might yield insight into the appropriateness of this string model with confined flux lines.

\section{Appendix}

In this appendix we give a proof of Lemma 6.

Let $\mathscr{H}_{\Lambda}$ be the subgroup of $C_{A}(\phi)$ generated by the elements $\partial c^{3}\left(\operatorname{dist}\left(c^{3}, \partial \lambda\right) \leqq 1\right)$ and assume $\mathscr{H}_{\Lambda} \neq C_{\Lambda}(\phi)$. Now for any $M \in C_{A}(\phi)$, define its radius by

$$
\operatorname{rad}(M)=\sup _{\substack{c^{1} \in c^{2} \\ c^{2} \in M}} \operatorname{dist}\left(X_{c^{1}}, X_{0}\right)
$$


where $X_{c^{1}}$ is the barycenter of $c^{1}$ and $X_{0}$ the center of $\Lambda$. Let $K(M)$ be the set of 1-cells $c^{1}$ in the closure of $M$ with $\operatorname{dist}\left(X_{c^{1}}, X_{0}\right)=\operatorname{rad}(M)$. Now among the manifolds in $C_{\Lambda}(\phi) \backslash \mathscr{H}_{\Lambda}$ with minimal radius, let $M_{0}$ be one which in addition has minimal $|K(M)|$. We will show that it is possible to find suitable $c_{j}^{3}(1 \leqq j \leqq n$; $2 n \leqq d(d-1))$ such that $M_{1}=M_{0}+\sum_{j=1}^{n} \partial c_{j}^{3}$ satisfies $\operatorname{rad}\left(M_{1}\right) \leqq \operatorname{rad}\left(M_{0}\right)$, and if $\operatorname{rad}\left(M_{1}\right)=\operatorname{rad}\left(M_{0}\right)$, then $\left|K\left(M_{1}\right)\right|<\left|K\left(M_{0}\right)\right|$. Since $M_{1} \in C_{\Lambda}(\phi) \backslash \mathscr{H}_{\Lambda}$ if $M_{0}$ is, this contradicts the choice of $M_{0}$, proving the lemma.

Now to find such $c_{j}^{3}$, let $c_{0}^{1}$ be an element of $K\left(M_{1}\right)$. Define $2 n$ to be the number of 2-cells $c_{1}^{2}, \ldots, c_{2 n}^{2}$, whose closure contains $c_{0}^{1}$. Since $\operatorname{dist}\left(X_{c_{0}^{1}}, X_{0}\right)=\operatorname{rad}(M)$, these 2-cells span pair wise different 2 planes.

Let $c_{j}^{3}$ be the 3 -cell spanned by $c_{2 j-1}^{2}$ and $c_{2 j}^{2}(1 \leqq j \leqq n)$. We claim these 3-cells have the desired properties.

Indeed, we first note that $c_{0}^{1}$ has now been removed, i.e. $c_{0}^{1}$ does not belong to the closure of any 2-cell in $M$. On the other hand, since the $c_{k}^{2}(1 \leqq k \leqq 2 n)$ belong to $M_{0}$, it is easy to see that any one cell $c^{1}$ belonging to the closure of some $c_{j}^{3}$ satisfies $\operatorname{dist}\left(X_{c^{1}}, X_{0}\right)<\operatorname{dist}\left(X_{c_{0}^{1}}, X_{0}\right)=\operatorname{rad}\left(M_{0}\right)$. Hence either $\operatorname{rad}\left(M_{1}\right)<\operatorname{rad}\left(M_{0}\right) \quad[$ if $\left.\left|K\left(M_{0}\right)\right|=1\right]$ or $\left|K\left(M_{1}\right)\right|<\left|K\left(M_{0}\right)\right|$ as was to be proved.

Acknowledgements. The author would like to thank Profs. H. Araki and H. Hironaka for their hospitality at RIMS and the Japan Society for the Promotion of Sciences for financial support. Discussions with T. Hara and G. Sterman have been very helpful.

\section{References}

[BDI] Balian, B., Drouffe, J.M., Itzykson, C.: Gauge fields on a lattice, gauge invariant Ising model. Phys. Rev. D 11, 2098-2103 (1975)

[C] Creutz, M.: Micro canonical Monte Carlo simulation. Phys. Rev. Lett. 50, 1411-1414 (1983)

[DFJ] Durhuus, B., Fröhlich, J., Jonsson, T.: Critical behaviour in a model of planar random surfaces. Phys. Lett 137 B, 93-97 (1984)

[F] Fröhlich, J.: The statistical mechanics of surfaces, preprint, to appear in the proceedings of the Sitges Conference 1984

[GJ] Glimm, J., Jaffe, A.: Quantum physics, a functional integral point of view. Berlin, Heidelberg, New York: Springer 1981

[G] Goto, T.: Relativistic quantum mechanics of two-dimensional mechanical continuum and subsidiary condition of dual resonance model Progr. Theor. Phys. 46, 1560-1569 (1971)

[KC] Kadanoff, L.P., Cerva, H.: Determination of operator algebra for the two-dimensional Ising model. Phys. Rev. D 3, 3918-2939 (1971)

[KT] Karowski, M., Thun, H.J.: Phase structure of systems of selfavoiding-surfaces, Phys. Rev. Lett. 54, 2556-2559 (1985)

[N] Nambu, Y.: Talk presented at the american physical society meeting in Chicago (1970), University of Chicago preprint EFI 70-07

[OS] Osterwalder, K., Schrader, R.: Axioms for Euclidean Green's functions. Commun. Math. Phys. 31, 83-112 (1973); 42, 281-305 (1975)

[OSe] Osterwalder, K., Seiler, E.: Gauge field theories on a lattice. Ann. Phys. 110, 440-471 (1978)

[Sch] Schrader, R.: On the Euler characteristic of random surfaces (to appear in J. Stat. Phys.) 
[Se] Seiler, E.: Gauge theories as a problem in constructive quantum field theory and statistical mechanics. Lecture Notes in Physics. Berlin, Heidelberg, New York: Springer 1982

[Si] Simon, B.: Functional integration and quantum physics. New York: Academic Press 1979

[SG] Sterling, T., Greensite, J.: Entropy of selfavoiding random surfaces. Phys. Lett. 121 B, 345-349 (1983)

[We] Wegner, F.J.: Duality in generalized Ising models and phase transitions without local order parameters. J. Math. Phys. 2259-2272 (1971)

[W] Wilson, K.: Confinement of quarks. Phys. Rev. D 10, 2445-2459 (1974)

Communicated by H. Araki

Received September 14, 1984; in revised form April 22, 1985

Note added in proof. As pointed out by the referee, the formulation in Sect. 2 is equivalent to the theory of dislocation variables ad discussed by Wegner [We] (see also [KC]), which provides the 3-dimensional extension of the famous Kramers-Wanniev duality transformation in the 2-dimensional Ising model. 